\title{
INFERTILIDADE: UM PROBLEMA DE SAÚDE PÚBLICA
}

\section{INFERTILITY: A PUBLIC HEALTH PROBLEM}

\author{
Daniel José da Silva ${ }^{1 *}$, Bárbara Pessoa de $\operatorname{Santana}^{2}$, Aarin Leal Santos ${ }^{1}$ (D) \\ ${ }^{1}$ Faculdade Sete de Setembro, Paulo Afonso, BA, Brasil. \\ ${ }^{2}$ Universidade Federal de Alagoas, Maceió, AL, Brasil. \\ *djsilva1010@live.com

\section{RESUMO}

Formar uma família ainda é um pensamento atual da sociedade. Contudo, para aqueles que desejam aumentar a progênie por meio de uma gestação, pode ter este desejo interrompido pela infertilidade e promover um choque avassalador. Dessa forma, esta problemática ganhou um espaço dentro do SUS, tornando-se um problema de saúde pública. A infertilidade é considerada quando o indivíduo possui chances mínimas de gerar um bebê. Portanto, a baixa fertilidade é um problema desde os tempos antigos e perpetuado, mesmo com visões diferentes, na contemporaneidade. Hoje o tratamento não é impedido pela falta de métodos, mas sim, pelo difícil acesso aos centros que se localizam nas grandes cidades. No decorrer dos anos, diversas leis e promulgações surgiram com a intenção de garantir o direito dos cidadãos ao acesso a um sistema de saúde, universal e integral, que cumpra com suas atribuições, desde a prevenção de uma gravidez indesejada à concepção, de modo natural ou não. Nesse sentido, este trabalho tem o propósito de esclarecer a compreensão sobre esta doença, as barreiras existentes e suas causas, bem como o conhecimento ao direito pela procura de centros que forneçam o tratamento.

Palavras-chave: Infertilidade. Saúde Pública. Sistema Único de Saúde.

\section{ABSTRACT}

Form a family it is still a current thinking of society. However, for those who wish to increase progeny through gestation, can have this desire interrupted by infertility and promote an overwhelming shock. In this way, this problem gained a space within the SUS, becoming a public health problem. The infertility is considered when the individual has minimal chances of generating a baby. Therefore, low fertility has been a problem since the ancient times and perpetuated, even with different visions, in the contemporaneity. Today the treatment is not impeded by the lack of methods, but by the difficult access to the centers that are located in the big cities. Over the years, various laws and promulgations have emerged with the intention of guaranteeing citizens' right to access to a health system, universal and integral, that fulfills its attributions, from the prevention of an unwanted pregnancy to conception, naturally or not. In this sense, this work has the purpose of clarifying the understanding about this disease, the existing barriers and their causes, as well as the knowledge to the right by the search of centers that provide the treatment.

Keywords: Infertility. Public Health. Unique Health System. 


\section{INTRODUÇÃO}

A infertilidade é considerada no momento em que há chances mínimas de gerar um bebê, ou seja, quando a fertilidade do casal é parcialmente baixa. Este é um problema na vida dos indivíduos afetados desde um tempo arcaico, principalmente na vida das mulheres. Estas eram potencialmente afetadas, marginalizadas, discriminadas e por vezes abandonadas e violentadas (JOPPERT JUNIOR et al., 2002). Com o avanço da medicina esse pensamento pode mudar e, portanto, o problema não mais subsiste na inexistência de tratamentos e sim na ausência de recursos terapêuticos acessíveis para todos os indivíduos prejudicados (OMBELET, 2011). Visto que os métodos se encontram em instalações públicas e a maioria está localizada nas grandes cidades, tornando-se assim, de difícil avesso os usuários (GERRITS, 2012).

Sendo uma das etapas mais importantes do ciclo da vida, ter filhos é considerado por muitos casais a realização de um sonho, "filhos trazem esperança à vida, é um afeto familiar" relatam as mulheres. Nessa lógica, a realização deste desejo pode ser interrompida pela presença da infertilidade, fato que diminui as chances de uma gravidez de modo natural. A incapacidade de ter filhos pode promover um choque avassalador sobre o modo de vida do casal, principalmente na mulher, com consequências sérias para o relacionamento conjugal (MIRANDA, 2005).

Nessa perspectiva, a infertilidade acaba sendo um problema de saúde pública, mas não é tratada com a devida importância. Pois além de ser considerada uma doença, o seu tratamento tornouse um direito a todos com afeição da evolução ética, política e sociocultural no mundo (PEREIRA, 2011; MAKUCH; BAHAMONDES, 2012). Tratamento este que deve ser disponível para toda a população, sem distinção (WHO, 2010a).

Em 2012, o termo "saúde" passou a ser sinônimo de "bem-estar físico, mental, espiritual e social". Essa trajetória deu início na década de 80 e 90, com o surgimento de leis e promulgações com o propósito de garantir esse conforto aos cidadãos (BRASIL, 1988; BRASIL, 1992; WHO, 2010a). Nesse mesmo intuito, em 1996 surgiu o modelo da Atenção Básica para melhorar a situação da saúde de modo a propiciar a prevenção de danos maiores (BRASIL, 2006; BRASIL, 2010). Da mesma forma, no final da década de 70 a Reforma Sanitária teve como proposta principal assegurar os direitos dos pacientes ao acesso à saúde sem discriminação e onde o contexto social, etário, regional e psicológico destes, é observado na sua totalidade (BRASIL, 1988; MATTOS, 2001; MATTOS, 2004). Portanto, da mesma maneira que é função do sistema de saúde prestar a assistência devida na prevenção de uma gravidez indesejada, é também sua atribuição oferecer meios para aqueles que anseiam por um filho por meio natural ou não (BRASIL, 2010).

Em razão da importância histórica e social da infertilidade, vê-se a necessidade de contribuir para um melhor entendimento sobre esta conduta para a população acadêmica e sociedade, trazendo informações sobre as suas causas e sobre o direito da busca por tratamentos em serviços de saúde pública. Dessa forma, proporcionar um esclarecimento sobre o que é a infertilidade, como deve ser tratada e quais dificuldades poder ser encontradas nesse caminho. Nesse seguimento deve-se advertir a população que a infertilidade deve ser posta como primeira instância, além de incentivar a busca por consultas para início do tratamento. Há cláusulas que esclarecem e de defendem que a infertilidade é um problema sociocultural e com consequência disso problemas psicológicos são presenciados. Com isso, há um esforço para minimizar e questionar todo esse conceito préestabelecido pela sociedade e formar para uma nova ressignificação sobre a infertilidade.

Assim, o objetivo deste trabalho é demonstrar como as pessoas lidavam e ainda lidam com a presença da infertilidade na sociedade e com o seu desenvolvimento ao longo do tempo, até se tonar um problema de saúde pública. Além de ampliar o conhecimento no âmbito da investigação e possíveis causas desta doença para um diagnóstico precoce, a disponibilidade do tratamento deve ser globalizada. Nesse sentido, também tem como intuito esclarecer que é um direito de todos a busca e a execução da terapia. A contrariedade nessa questão se faz pela falta de assistência dada para a resolução desta problemática e pela escassa disponibilidade de vagas nesses centros. Tais 
modificações certamente promoveriam a redução da complexidade, de forma a trazer uma maior acessibilidade a toda população.

\section{METODOLOGIA}

Este trabalho é oriundo de uma pesquisa de revisão de literatura sobre o tema "Infertilidade: um problema de saúde pública". Para se levantar dados sobre o campo de interesse, foram feitas pesquisas que enfatizaram o histórico da infertilidade em geral, direcionado à prática aplicada à espécie humana, as barreiras ético-culturais relacionadas à sua utilização, métodos e técnicas utilizadas dentro dos recursos terapêuticos.

Os conteúdos analisados foram organizados de acordo com o tema pré-estabelecido. Do mesmo modo, todas as informações contidas no material lido foram ordenadas e utilizadas a partir da consideração de sua relevância.

A coleta de dados foi realizada por base de publicações referenciadas em revistas e nos sites de pesquisas, como a National Library of Medicine (PUBMED); Literatura Latino Americano e do Caribe em Ciências Sociais e da Saúde (LILACS) e Scientific Electronic Library Online (SCIELO); sites que têm disponíveis os conteúdos desejados. Entre as buscadas, todas entre os anos de 1984 a 2016, permitindo conhecimentos antigos e os mais recentes sobre o referido tema, abordando assuntos que nós permitimos alcançar informações e como foco, uma resposta para a temática. Com base nos idiomas, os conteúdos foram utilizados na língua inglesa e português brasileiro.

As seleções dos dados foram traçadas inicialmente pela busca de temas e títulos. Em seguida foram feitas leituras breves em seus resumos e objetivos, aprimorando uma maior seletividade dos materiais encontrados. E por fim, uma análise mais precisa foi abordada em todos os materiais selecionados, priorizando cada detalhe necessário para a elaboração do trabalho, resultando na utilização de 46 materiais, entre eles artigos, resoluções e leis, com o objetivo de trazer informações e respostas para a problemática abordada.

\section{DESENVOLVIMENTO}

\section{Infertilidade: A busca pela superação dos limites}

A infertilidade é um transtorno para os indivíduos afetados, antigamente vistos como amaldiçoados e rejeitados pela sociedade. Os considerados inférteis viviam sozinhos, excluídos, perdiam a sua posição social e sofriam com a discriminação daqueles considerados férteis, os quais eram bem vistos na comunidade. Para algumas culturas, mesmo hoje em dia, mulheres têm a obrigação de gerar filhos, aquelas que não aderem estão sujeitas a serem abandonadas por seus maridos, alienadas e até mesmo violentadas (JOPPERT JUNIOR et al., 2002; WHO, 2010a).

Hoje com evolução da ciência médica nas últimas décadas, surgiram meios, a Reprodução Assistida (RA), que oferecem tratamentos que aumentam as chances de sucesso de uma gestação para aqueles que buscam uma gestação. Dessa forma, o desejo de filhos não mais reside na inexistência de métodos (OMBELET, 2011).

No momento presente a infertilidade é considerada um problema de saúde pública e se tornou um direito de todos com afeição da evolução ética, política e sociocultural no mundo. O tratamento da infertilidade deve ser globalizado e assimilado para a identificação da necessidade de cada caso específico. Com isso, as variadas técnicas de infertilização são direcionadas a cada caso, o que evita danos futuros, como a utilização de métodos não necessária (WHO, 2010b; MAKUCH; BAHAMONDES, 2012).

Infertilidade é uma doença do sistema reprodutor masculino ou feminino definida pelo fracasso em conseguir uma gravidez após 12 meses ou mais de relações sexuais regulares sem proteção (OMS, 2020). infertilidade pode ser classificada em primária, quando há dificuldade de gerar a primeira gravidez, e secundária quando há problemas em uma segunda gestação (SOUZA, 2008). 
Mesmo com avanços, o pensamento antigo ainda se faz presente, pessoas não procuram ajuda médica por presumir que a fertilidade seria um carma, uma sina. Do mesmo modo, o medo do diagnóstico faz com que estas não busquem soluções, e por fim impossibilitam o tratamento em si. $\mathrm{O}$ não ter filhos pode acarretar consequências severas tais como o isolamento, que deve ser entendido como um pedido de socorro (VAN; BOS, 2009).

\section{Infertilidade conjugal}

Muitos casais consideram que ter filhos é uma das mais importantes etapas do ciclo da vida. Algumas mulheres, por exemplo, relatam que "filhos trazem esperança à vida, é um afeto familiar". Entretanto, a realização deste desejo nem sempre acontece de forma natural. Em muitos casos, os planos podem estar impedidos, ocasião esta em que se diz haver a infertilidade conjugal. A incapacidade de ter filhos pode promover um choque avassalador sobre o modo de vida do casal, principalmente na mulher, com consequências sérias para o relacionamento conjugal (MIRANDA, 2005).

Casais que não conseguem uma gravidez durante um ano, com relação sexual e sem o uso de contraceptivos, são considerados inférteis pela OMS. (CHEDID, 2000). As causas da infertilidade não estão relacionadas à apenas o sexo feminino, mas $30 \%$ dos casos estão relacionados aos homens (tabela 1) e é decorrente também de diversos fatores como a idade, o estilo de vida, contaminação por radiações químicas e alteração hormonal de ambos os sexos (FEBRASGO, 1997).

Tabela 1 - Causas da infertilidade conjugal

\begin{tabular}{ll}
\hline Causas & $\%$ \\
\hline Masculino & 30 \\
Feminino & 35 \\
Desconhecido & 15 \\
Combinação conjugal & 20 \\
\hline
\end{tabular}

Fonte: Souza e Vitorino (2008).

Nessa perspectiva, o planejamento familiar é fundamental, e, pensando nisso, o Presidente da República, sobre o Congresso Nacional, sancionou a Lei 9.263, de 12 de janeiro de 1996, do planejamento familiar, assegurando ser este um direito igualitário de todo cidadão, na mesma medida em que faz parte da totalidade de procedimentos pertinentes à atenção da mulher, do homem ou do casal. O Art. $9^{\circ}$ do Capítulo I afirma:

“Art. $9^{\circ}$ Para o exercício do direito ao planejamento familiar, serão oferecidos todos os métodos e técnicas de concepção e contracepção cientificamente aceitos e que não coloquem em risco a vida e a saúde das pessoas, garantida a liberdade de opção. Parágrafo único. A prescrição a que se refere o caput só poderá ocorrer mediante avaliação e acompanhamento clínico e com informação sobre os seus riscos, vantagens, desvantagens e eficácia" (BRASIL, 1996).

Para isto, a investigação deste problema consiste em uma análise conjugal, onde as características conjuntas e individuais são avaliadas (Figura 1) (GERRITS, 2012). A saúde do casal é um dos parâmetros mais importantes a ser analisado, por isso eles devem estar livres de doenças infecciosas, oncológicas e outras enfermidades como o HIV, sífilis, hepatites B e C (FEBRASGO, 1997).

A anamnese é o primeiro passo em uma análise conjugal. Toda a vivência dos conjugues é levada em consideração como o modo vida, a frequência do ato sexual sem contraceptivos, relatos sobre a infertilidade e se houve tratamento citado e quais exames foram realizados, se há histórico hereditário, se o ciclo menstrual funciona regulamente, se houve gravidez anterior, se já sofreu aborto 
espontâneo ou provocado, se há presença de filhos do casal ou em relacionamento anteriores, ou seja, todas as informações devem ser averiguadas (FEBRASGO, 1997).

Figura 1 - Direções para uma investigação de problemas inférteis

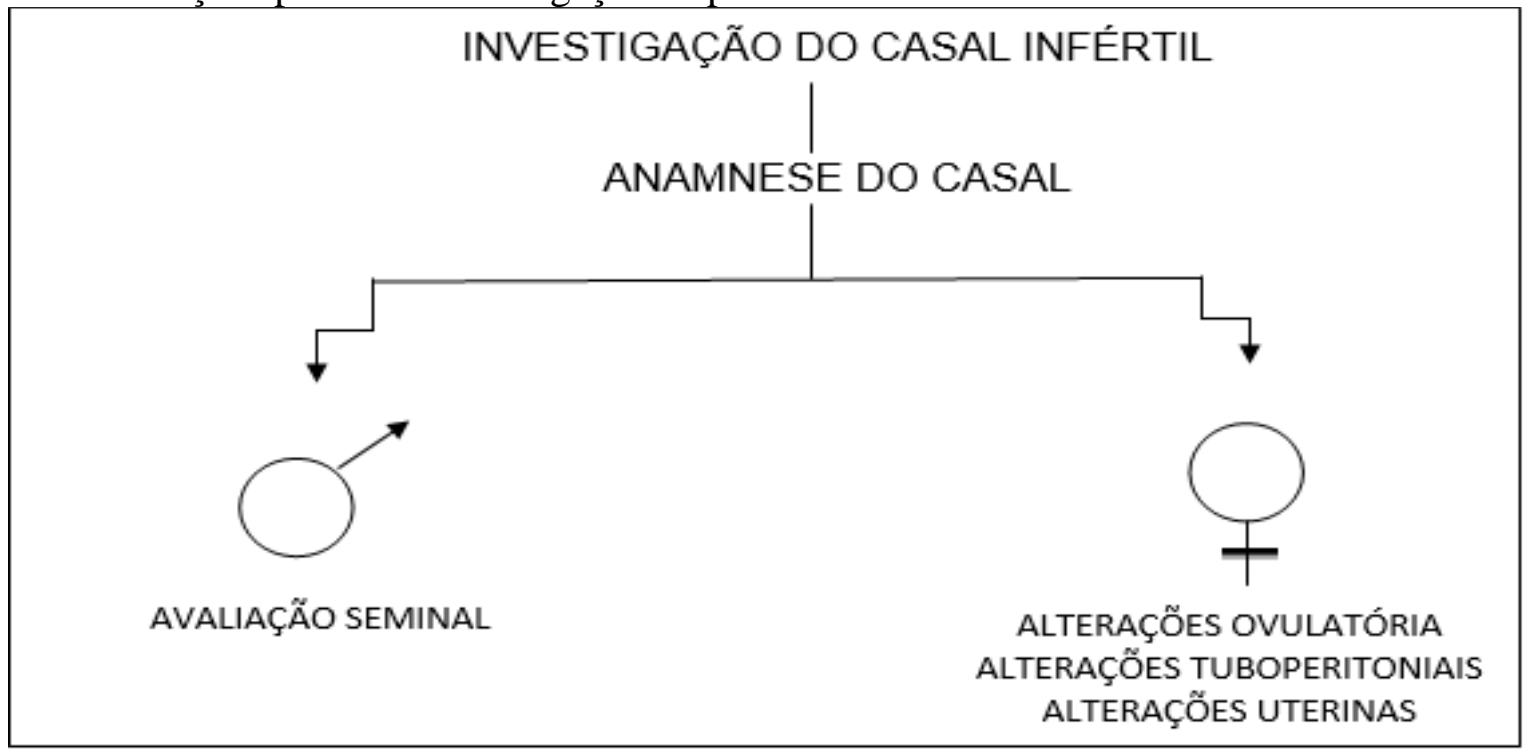

Fonte: Almeida et al. (2018).

\section{Infertilidade feminina}

No período do regime militar, onde o tema "mulher" era discutido nos seus mais diversos aspectos, manifestos feministas surgiram com muita força e estimularam o âmbito da saúde. Em 1975 foi lançado o "Saúde Materno-Infantil" um programa com o intuito de organizar a família e reduzir o índice de mortalidade infantil (MOURA; SILVA, 2004). Com o passar dos anos, o Ministério da Saúde - MS criou o programa de Atenção Integral à Saúde da Mulher - PAISM (BRASIL, 1984). Este programa destinado à saúde primária tinha como objetivo promover soluções para atender as necessidades da saúde da mulher, assim, esse modelo envolveria uma vasta busca para o atendimento integral, como a avaliação ginecológica, pré-natal, parto e programas educacionais como as DST's, os contraceptivos e o câncer cérvico-uterino e de mama (OSIS, 1998; SOUZA, 2004).

Neste mesmo contexto, acredita-se que a fecundidade e feminidade andam juntas, e apesar de ainda surgirem entraves, o sonho de ser mãe nunca foi descartado da maioria da população feminina o que se torna uma busca mais acentuada por soluções (AVELAR et al., 2000). Segundo Yin (1987), o fato da presença da infertilidade na mulher afeta sua autoestima, comprometendo a sua saúde emocional que a lhe tornam vagas, insegurança e que pode afetar a vida sexual e conjugal (YIN, 1987). O caminho para a descoberta da infertilidade feminina é bem complexo, por existirem diversos fatores relacionados a esse problema. No entanto, para ocorrer uma gravidez é preciso que o sistema reprodutor feminino esteja em perfeitas condições (SOUZA, 2008).

Outro ponto a ser analisado é a inserção das mulheres no mercado de trabalho, motivo pela qual as mesmas adiam o "aumento da família" e com isso deixam para pensar numa possível gravidez com idade avançada. Segundo Lock (2000), a fertilidade da mulher diminui com a idade: mulheres com 30 anos têm a sua fertilidade de $90 \%, 40$ a 44 anos tem $62 \%$ e a partir dos 45 apresentam apenas $14 \%$ da sua fertilidade. Há estudos que descrevem que mulheres na idade dos 50 anos necessitam de mais estímulos hormonais para que consigam a maturação dos seus folículos, outro motivo seria o índice de abortos, mulheres com idade mais avançada apresentam tendência para abortos com mais frequência (LOCK, 2000).

A investigação primária de problemas, que visa descobrir qual a dificuldade da gravidez inclui a investigação do ciclo menstrual, visto que a sua ausência pode implicar problemas ovulatórios. A inexistência do ciclo (amenorreia) ou a menstruação irregular (oligomenorreia) resultam na falta e na 
baixa produção ovulatória respectivamente, circunstância esta, presente em casos de infertilidade. Exames como a dosagem de progesterona, FSH, LH, estradiol, prolactina, exames por imagens e laboratoriais auxiliam para um bom diagnóstico em problemas na ovulação e, em outros acontecimentos podem ser solicitados diversos exames (SOUZA, 2008).

Os problemas ovulatórios podem ser classificados em grupos: No primeiro grupo são as causas de problemas na maturação folicular. $O$ segundo grupo está relacionado a problemas metabólicos, como a síndrome dos ovários policísticos (SOP). No terceiro grupo, os problemas são a nível ovariano, os hormônios são encontrados em níveis alterados, como por exemplo, estrogênios em menor quantidade e as gonadotrofinas em uma quantidade elevada, nesse caso, há possibilidade de corrigir falência ovariana congênita ou adquirida, em caso de aplicações de quimioterapia (FEBRASGO, 1997).

$\mathrm{Na}$ fecundação, as tubas uterinas são de alta importância, é por ela que milhares de espermatozoides percorrerão até o encontro com o óvulo, sendo que alguma alteração presente decorrente de infecções por vírus e bactérias transmitidas por relação sexual, o uso do Dispositivo Intrauterina (DIU), processo cirúrgico de apendicite supurada ou abortos, dificultam ou até mesmo impossibilitam a concepção (MAIA, 2009).

No diagnóstico de obstrução tubária é utilizado a histerosalpingograma, um exame que possibilita a visualização anatômica das tubas uterinas com o auxílio de contrastes. Deve ser aplicado entre o $7^{\circ}$ e o $10^{\circ}$ dia do ciclo menstrual, exceto se houver a presença de algum processo inflamatório na área genital. Nessa situação ocorre a avaliação de qualquer anormalidade (KARASICK, 1991).

Problemas uterinos são mais frequentes e merecem um cuidado exclusivo. Composto por colo e corpo uterino, o útero tem uma grande importância na sobrevivência do óvulo fecundado. Os exames rotineiros, como a ultrassonografia e o Papanicolau, tem a capacidade de diagnosticar anormalidades como a endometriose, câncer do colo do útero, miomas e pólipos (ASRM, 2012).

Embora não seja muito solicitado, a utilização do exame pós-coito possibilita o diagnóstico dos possíveis problemas em gerar uma gravidez que não é perceptível em exames por imagem. O exame é baseado na investigação do $\mathrm{pH}$ e na presença de bacilos no muco cervical - secreção que ajuda na passagem dos espermatozoides até óvulo. O teste relata problemas no muco cervical ou direciona a uma análise seminal do parceiro (OEI; HELMERHORST; KEIRSE, 1995).

\section{Infertilidade Masculina}

As mulheres ocupam o lugar das mais recriminadas e acusadas pela não procriação, embora os homens sejam predispostos à infertilidade tanto quanto elas C (RUTSTEIN; SHAH, 2004). Em virtude de que o fator masculino é responsável por 30\% das vezes em que uma gestação não pode acontecer, a investigação das causas da infertilidade masculina inicia-se pela anamnese, com exames físicos e complementares realizados por especialistas em reprodução masculina. A anamnese busca informações sobre a vida do paciente: idade, profissão, se já houve contato com infecções, presença de doenças sexualmente transmissíveis, presença de filhos, se há relatos de problemas hereditários, hábitos sexuais, etc. Todos estes parâmetros ajudam a diagnosticar possíveis distúrbios de fertilidade (SOUZA; VITORINO, 2008).

Conforme já apontamos, a estimativa do homem em relação a infertilidade é de aproximadamente $30 \%$ em problemas reversíveis ou irreversíveis, como idade, ejaculação retrógrada, tumor testicular, hipogonadismo, varicocele, síndrome de Klinefelter, criptorquidia e casos idiopáticos (SOUZA, 2008). No homem, o problema com a idade chega aos 40 e 50 anos, nesse sentido atinge diretamente na frequência do ato sexual, contudo não interfere na produção seminal, mas o problema é presenciado na morfologia e motilidade espermática, índices de extrema importância em uma fecundação, com isso, a idade é um fator conjunto na dificuldade de uma gestação (FEBRASGO, 1997).

O exame físico tem como função avaliar o hábito corporal e as características sexuais. Durante a avaliação testicular, deve ser feita a análise de sua consistência e tamanho, pois a sua irregularidade 
afeta diretamente a produção espermática. A presença da varicocele pode ser detectada no exame físico (SHEFI; TUREK, 2006). A varicocele, ou "bolsa de varizes", é definida como uma dilatação venosa e sinuosa referente às veias espermáticas, causada por um refluxo sanguíneo testicular aumentando a temperatura escrotal promovendo alterações no crescimento testicular e na produção espermática (PASQUALOTTO; PASQUALOTTO, 2006).

Outros procedimentos complementares trazem direções no fechamento do diagnóstico da infertilidade. O espermograma é o método mais solicitado, não necessariamente com uma espermocultura, porém a sua requisição pode ser útil (FEBRASGO, 1997). O espermograma tem como finalidade a análise seminal com diagnóstico de problemas no sistema reprodutor e da condição fértil. Solicitado quando há uma incapacidade de uma gestação que pode estar relacionado a problemas hereditários, condições físicas e problemas imunológicos do paciente (PASQUALOTTO; PASQUALOTTO, 2006).

\section{Infertilidade: um problema de saúde pública}

De acordo com a Constituição Federal de 1988, o Art. 196., prediz que o acesso à saúde, além de ser um direito de todos e dever do estado, deve permitir o acesso universal e igualitário à todas as suas cláusulas, sobretudo suas ações e serviços para promoção, proteção e recuperação da sociedade (BRASIL, 1988). Em 1992, o Conselho Federal de Medicina (CFM) publicou a Resolução 1.358, documento que considera a infertilidade humana como um problema de saúde, com consequências médicas e psicológicas (CFM, 1992). Com isso, a resolução também defende que todos os tratamentos possíveis devem recomendar a utilização da Reprodução Assistida (RA), utilizando-a apenas em último caso (BRASIL, 1988; SAMRSLA et al., 2007). Em 2010, uma publicação da Organização Mundial da Saúde - World Health Organization (OMS - WHO) demonstrou que 15\% da população mundial sofre com a infertilidade (WHO, 2010b). Segundo estudos e pesquisas realizadas no Brasil, há cerca de 5 milhões de casais inférteis (SANTOS; UGÁ; PORTOS, 2008).

Contudo, em pleno século XXI, a infertilidade não é priorizada como deveria em países com poucos recursos, por mais que este impasse tenha se tornado um problema de saúde pública. Nessa perspectiva, surgem empecilhos que dificultam a resolução de vários problemas, entre eles estão o descaso por parte do governo no investimento para solucionar este problema, bem como, a desigualdade evidente na sociedade para o acesso às técnicas de tratamento, onde muitos recursos ainda não estão disponíveis e quando se encontra presente é de difícil acesso (MAKUCH; PETTA; OSIS, 2010; OMBELET; VAN, 2012).

A literatura corrobora com a ideia de que a presença da infertilidade em homens e/ou mulheres traz consequências psicológicas na busca para a concretização do sonho de ter um bebê (MAKUCH; BAHAMONDES, 2012). Visto que o impacto deste transtorno costuma ser superior nas mulheres do que nos homens. São elas as que mais exprimem a sua dor e refletem sobre o incômodo, o que resulta em grande ansiedade e no declínio da sua autoestima. A literatura também comprova que a presença desta barreira, na vida da mulher, resulta em duas vezes mais depressão quando comparada com a população em geral. Nesse mesmo contexto, os homens também sofrem devido à dificuldade em expressar suas emoções, podendo torná-los estressados e conturbar a vida conjugal (FÉLIS; ALMEIDA, 2016). Devido a relevância dessa problemática, e as consequências sobre a saúde e o bem-estar dos envolvidos, esta doença precisa urgentemente ser plenamente considerada na saúde pública (GRADVOHL; OSIS; MAKUCH, 2013).

Em uma entrevista com autoridades de saúde a nível estadual e municipal, a existência do tratamento da infertilidade, porém estas autoridades possuem esclarecimentos diferentes do mesmo problema, contudo o reconhecem como um contratempo. Nessa perspectiva, uma parte relatou que não há presença dos serviços, pois a administração de saúde não tem compromisso em fornecer este tratamento. Apesar disso, a outra parte descreveu que na região em que se reside, o modelo ainda não se faz presente dentro do Sistema Único de Saúde (SUS). Com isso, a explicação popular mais óbvia dessa inexistência é dada pela omissão dessa complicação através dos estados e municípios, os quais 
alegam um baixo investimento para a resolução dessa necessidade, assim como a escassez de profissionais específicos nessa área. Mesmo assim, com todas essas objeções a busca por tratamentos têm se tornado possível (MAKUCH; BAHAMONDES, 2012).

Ainda sobre a entrevista citada acima, alguns centros que fornecem esse tratamento foram visitados e entre todos eles apenas um se faz presente na região analisada, enquanto os outros se encontram distante da localização, porém todos os cinco visitados forneciam total procedimento dentro do SUS. Os profissionais que fornecem serviços nesses centros, em conjunto com os pacientes, também foram entrevistados. Nessa entrevista, relataram que a porta de entrada para o início do tratamento era feita por meio de encaminhamento médico na Unidade Básica de Saúde (UBS) ou de uma consulta diretamente no centro de tratamento, o que torna o direito limitado aos procedimentos, e ainda mais quando há necessidade de outros procedimentos invasivos (MAKUCH; BAHAMONDES, 2012).

Nessa mesma lógica, Freitas et al. (2008), na sua pesquisa sobre esse mesmo assunto, nos revela que no Brasil há 177 centros que fornecem técnicas de reprodução assistida para casais inférteis, entre eles, seis centros suprem a necessidade da população, com o tratamento completamente gratuito, três liberam o tratamento, mas o custo dos medicamentos é de responsabilidade dos pacientes e por fim, outros fornecem apenas a investigação e o diagnóstico da infertilidade, sem o acompanhamento pós-diagnóstico. Além destes, há poucos centros que oferecem o tratamento gratuito, todavia os mesmos estão localizados nas capitais e grandes cidades, dificultando o acesso a aqueles que residem em cidades do interior (FREITAS; SIQUEIRA; SEGRE, 2008; MAKUCH; PETTA; OSIS, 2010).

Além disso, a fila de espera dificulta a conquista rápida de uma vaga para o início do tratamento, uma vez que a sua demora na busca por tratamentos e muitas vezes o atraso no diagnóstico, expõe um embate desfavorável ao tratamento e a pesquisa por soluções para este problema (BRASIL, 1988). Dentro do Serviço Público, esse atraso se torna ainda maior e com mais frequência, sendo que isso se dá devido a demanda ser superior a quantidade de vagas ofertadas pelo sistema. Neste caso, os pacientes levam de meses a cinco anos para iniciar o tratamento. Toda essa demora reflete muitas vezes em ansiedade, desânimo e frustração e leva a desistência por muitos casais, como já citado anteriormente (DENTILLO, 2012; FÉLIS; ALMEIDA, 2016).

\section{A infertilidade dentro da Atenção Básica à Saúde}

O significado de saúde teve uma nova definição pela Organização Mundial de Saúde. O que era conhecido como "a ausência de doença" passou a ser sinônimo de "bem-estar físico, mental, espiritual e social" (WHO, 2010a). E com esse mesmo pensamento, há muito tempo atrás, surgiram leis e promulgações como a CF de 1988 e CFM de 1992 para assegurar as primícias, ao mesmo tempo em que as implicações são observadas, diagnosticadas e superadas (BRASIL, 1988; CFM, 1992).

O modelo da Atenção Básica surgiu em 1996, com a intenção de melhorar a situação da saúde. Nesse sentido, promover um atendimento universal e integral, ao proporcionar direito e livre acesso à assistência. Em consequência disso, favorecer o desenvolvimento, o acolhimento e propiciar a prevenção de agravos. Da mesma forma, possibilitar o diagnóstico, tratamento e reabilitação da saúde com tecnologias de alta e baixa complexidade, no plano individual e coletivo da população que busca por esse tipo de atendimento (BRASIL, 2006; BRASIL, 2010).

O movimento da Reforma Sanitária, no final da década de 70, foi marcado por garantir que o paciente fosse observado como um indivíduo completo, e não apenas como um doente ou um sistema (MATTOS, 2001). A universalidade e integralidade, em conjunto, tiveram consequências na modificação do Sistema de Saúde. Onde a primeira deu o direito a todos os cidadãos brasileiros ao acesso as ações de saúde, sem qualquer discriminação (MATTOS, 2004). E o segundo termo ainda não é visto de forma explícita na diretriz, a expressão utilizada é "atendimento integral", uma das concepções do SUS, onde o contexto social, etário, regional e psicológico do paciente deve ser levado 
em consideração e o sistema de saúde tem por dever atender a demanda e a necessidade do indivíduo (BRASIL, 1988).

Nesse caso, a integralidade tem um olhar extenso na busca por soluções para minimizar e solucionar certos transtornos do indivíduo, assegurando o cidadão sobre o seu direito de acesso a todos os níveis de complexidade, do baixo ao alto. Dessa forma, se torna um suporte para que haja uma ótima prestação de serviços para a saúde (FONTOURA; MEYER, 2006).

O sistema de saúde tem como função auxiliar na prevenção de gravidez indesejada ao ofertar contraceptivos, da mesma forma que tem a incumbência em oferecer recursos para aqueles que planejam ter filhos, seja por meio natural ou não. Mesmo que o princípio da portaria $\mathrm{n}^{\mathrm{o}} 426 / 2005$ determine a assistência à infertilidade dentro do Sistema Único de Saúde, ainda há muitos empecilhos para efetivação desse ato. Dessa forma, os indivíduos acometidos por este diagnóstico veem na Atenção Básica, um importante auxílio, pois é a porta de entrada principal para a busca de tratamento. $\mathrm{O}$ espaço se transformou em um ambiente onde a orientação é fundamental e significativa, ao envolver diagnóstico e intervenção (BRASIL, 2010).

Os autores Joppert Junior et al. (2002), e Ombelet (2011), trazem conceitos em suas matérias de forma evolutiva. O primeiro autor aborda como a infertilidade era vista em épocas passadas. Assim, em seu conteúdo é observado relatos em que as mulheres tinham por obrigação de gerar filhos e aqueles, homens e mulheres, inférteis eram descriminados diante da sociedade, pois os mesmos eram considerados amaldiçoados. Contudo, o segundo autor, aborda a visão diante a infertilidade hoje em dia. Para este, as ciências médicas evoluíram. Portanto, hoje, há diversas abordagens tecnológicas em diagnóstico e tratamento eficazes para se conceber um bebê.

A partir disto, a World Health Organization (2010), diz que a infertilidade é considerada um problema de saúde pública e que o seu tratamento deve ser globalizado, assimilado para a identificação das suas causas. No Art. 196 da Constituição Federal de 1988 relata que "o acesso à saúde, além de ser um direito de todos e dever do estado, deve permitir o acesso universal e igualitário a todas as suas cláusulas, sobretudo suas ações e serviços para promoção, proteção e recuperação da sociedade".

Nessa sequência, Dentillo (2012), Félis e Almeida (2016) abordam o atraso do atendimento no Sistema Público devido a sua demanda diante a quantidade de vagas prestadas, podendo levar de meses a anos para o início do tratamento.

\section{CONSIIDERAÇÕES FINAIS}

O objetivo deste trabalho, além de analisar a oferta de recursos terapêuticos para a infertilidade no âmbito dos serviços públicos, teve como intuito explorar conhecimentos informativos de conteúdos que circundam este dilema. Neste caso, a assimilação dos transtornos causados pela existência dessa doença e o direito à busca por tratamentos nesses serviços. Uma vez que os assuntos aqui debatidos visam expor o real cenário de como essa demanda tem sido atendida na saúde pública brasileira.

Além disto, a partir da abordagem histórica realizada, ficou evidente que em tempos arcaicos a tradição familiar tinha um grande valor, e aqueles que sofriam com esse transtorno eram abandonados e isolados, o que provocava a perda do seu lugar na sociedade. Visto que as crenças e costumes das culturas vigentes e predominantes tinham poder e ação sobre estas pessoas, o que se dava pela falta do reconhecimento deste problema como um fator de saúde. Passado o tempo, a vontade de seguir com a tradição familiar ainda se faz presente e, hoje, o problema com a infertilidade ainda causa danos psicológicos naqueles que buscam a paternidade/maternidade. Ainda, com uma maior prevalência nas mulheres, pois além da falta de disseminação de informações a respeito do problema, parte significante da oferta dos serviços que visam sanar este distúrbio, acaba sendo falha devido à falta de um maior interesse governamental, apesar da busca pelo tratamento ter se tornado possível. 
No entanto, a infertilidade ganhou espaço dentro do SUS, foi reconhecida como uma doença e que seu tratamento deve ser prestado nos serviços públicos. Porém, como dito, o espaço ainda se encontra carente, uma vez que a União, juntamente com os governos municipais e estaduais, principais fontes de investimento, tem como prioridade o tratamento de outras patologias. Esta informação evidencia um descaso na priorização deste problema.

A distribuição dos centros ainda é escassa e a maioria está localizada nas grandes cidades, dificultando o seu acesso. São poucos os que oferecem o tratamento completo, e, em alguns, o serviço é oferecido parcialmente, apenas para consulta e finalizando no diagnóstico. Já em outros, para a realização dos procedimentos o paciente é responsável pela compra de medicamentos.

O resultado obtido desse trabalho ajuda no conhecimento fidedigno da posição da infertilidade dentro do sistema público, torna compreensível os procedimentos de investigação para um diagnóstico dessa patologia e transparece todo empecilho que ainda há em sua busca.

Com esta abordagem, conclui-se que a infertilidade é de fato um problema com sérios reflexos na vida daqueles que estão acometidos desta condição. Uma vez que, sendo considerado um problema de saúde pública e tendo o seu tratamento garantido por direito, ainda há a falta de priorização e investimentos a respeito da oferta dos métodos necessários. Por fim, todos esses empecilhos ressaltam a barreira que é criada para quem procura e precisa do acesso.

\section{REFERÊNCIAS}

AVELAR, C. M. et al. Emoção e infertilidade. Reprodução \& Climatério, v. 15, n. 4, p. 203-205, 2000.

BRASIL. Ministério da Saúde. Política Nacional de Atenção à Saúde da Mulher, 1984.

BRASIL. Diário Oficial da União Conselho Federal De Medicina. Resolução CFM n ${ }^{\circ}$ 1.358/1992. Publicada no D.O.U., 19 de novembro de 1992, Seção I, p.16053, 1992.

BRASIL. Casa Civil. Lei no 9.263, de 12 de janeiro de 1996. Brasília, DF, 1996.

BRASIL. Constituição da República Federativa do Brasil - 1998. São Paulo: Revista dos Tribunais., 1998.

BRASIL. Ministério da Saúde. Secretaria de Atenção à Saúde. Departamento de Atenção à Saúde. Política Nacional de Atenção Básica. - Brasília: Ministério da Saúde, 2006.

BRASIL. Ministério da Saúde. Secretaria de Atenção à Saúde. Departamento de Atenção Básica. Saúde sexual e saúde reprodutiva/Ministério da Saúde, Secretaria de Atenção à Saúde. (Série A. Normas e Manuais Técnicos) (Cadernos de Atenção Básica, n. 26). Departamento de Atenção Básica. - Brasília: Ministério da Saúde. 300p. 2010.

BRASIL. Constituição da República Federativa do Brasil - 1998. Seção II - Da saúde. Art 196. p. 118-119. Brasília: Senado Federal, Coordenação de Edições Técnicas, 2016.

CONSELHO FEDERAL DE MEDICINA. Resolução n. 1358 de 1992. Adota as normas éticas para a utilização das técnicas de reprodução assistida, anexas à presente resolução como dispositivo deontológico a ser seguido pelos médicos. Diário Oficial da República Federativa do Brasil, Brasília, Seção I. 19 de nov. 1992, p. 16.053.

DENTILLO, D. B. Cresce demanda por tratamento de infertilidade, mas o acesso é ainda caro e seletivo. Ciência e Cultura, v. 64, n. 4, p. 10-11, 2012. 
CHEDID, S. Infertilidade. 2. ed. São Paulo: Contexto, 2000.

PRACTICE COMMITTEE OF THE AMERICAN SOCIETY FOR REPRODUCTIVE MEDICINE. Endometriosis and infertility: a committee opinion. Fertil Steril, v. 98, n. 3, p. 591-598, 2012.

FÉLIS, K. C; ALMEIDA, R. J. Perspectiva de casais em relação à infertilidade e reprodução assistida: uma revisão sistemática. Reprodução \& Climatério. v. 31, n. 2, p. 105-111, 2016.

FREITAS, M.; SIQUEIRA, A. A. F.; SEGRE, C. A. M. Avanços em Reprodução Assistida. Revista Brasileira Crescimento Desenvolvimento Humano, v. 18, n. 1, p. 93-97, 2008.

FONTOURA, R. T.; MEYER, C. N. Uma breve reflexão sobre a integralidade. Revista Brasileira de Enfermagem, v. 59, n. 4, p. 532-537, 2006.

FEBRASGO. Infertilidade conjugal. In: Manual de Orientação Reprodução Humana, 1997. 93p.

GERRITS, T. Biomedical infertility care in low resource countries: Barriers and Access. Factore, Views e Vision is ObGyn, p. 1-6, 2012.

GRADVOHL, S. M. O.; OSIS, M. J. D.; MAKUCH, M. Y. Estresse de homens e mulheres que buscam tratamento para infertilidade. Revista Brasileira de Ginecologia e Obstetrícia, v. 35, n. 6, p. 255-261, 2013.

JOPPERT JUNIOR, A. et al. Reprodução assistida aspectos históricos. Intertem@s, v. 4, n. 4. p. 1$18,2002$.

KARASICK, S. Hysterosalpingography. Radiologia Urologia, v. 13, p. 67-73, 1991.

LOCK, S. E. Contracepção, infertilidade e aborto. $O$ cuidado em enfermagem materno. 5. ed. Cap. 7. Porto Alegre: Artmed, 2000.

MAIA, H. Histerosalpingografia: introdução ao estudo da radiologia ginecológica. Salvador: EDUFBA, 2009.

MAKUCH, M. Y.; BAHAMONDES, L. Barriers to access to infertility care and assisted reproductive technology within the public health sector in Brazil. Factore, Views e Vision is ObGyn, v. 4, n. 4, p. 221-226, 2012.

MATTOS, R. A. A integralidade na prática (ou sobre a prática da integralidade). Cadernos de Saúde Pública, v. 20, n. 5, p. 1411-1416, 2004.

MATTOS, R. A. Os sentidos da integralidade: algumas reflexões acerca de valores que merecem ser defendidos. Instituto de Medicina Social: ABRASCO, Rio de Janeiro: UERJ. 2001

MIRANDA, F. E. A infertilidade feminina na pós-modernidade e seus reflexos na subjetividade de uma mulher. Psicologia em Revista, v. 11, n. 18, p. 271-273, 2005.

MOURA, E. R. F.; SILVA, R. M. Informação e planejamento familiar como medidas de promoção da saúde. Ciência e Saúde Coletiva, v. 9, n. 4, p. 1023-1032, 2004. 
MAKUCH, M. Y.; PETTA, C. A.; OSIS, M. J. Low priority level for infertility services within the public health sector: a brazilian case study. Human Reproduction, v. 25, n. 2, p. 430-435, 2010.

ORGANIZAÇÃO MUNDIAL DE SAÚDE. Infertility, p. 1-5. 2020.

OSIS, M. J. M. D. PAISM: um marco na abordagem da saúde reprodutiva no Brasil. Cadernos de Saúde Pública, v. 14, n. Supl. 1, p. 25-32, 1998.

OMBELET, W. Global access to infertility care in developing countries: a case of human rights, equity and social justice. Factore, Views e Vision is ObGyn, v. 3, n. 4, p. 257-266, 2011.

OEI, S. G.; HELMERHORST, F. M.; KEIRSE, M. J. When is the post-coital test normal? A critical appraisal. Human Reproduction, v. 10, n. 7, p. 1711-1714, 1995.

OMBELET, W.; VAN BALEM, F. Future Perspectives. Factore, Views e Vision is ObGyn, p. 8790, 2012.

PASQUALOTTO, E. B.; PASQUALOTTO, F. F. Espermograma e testes de função espermática. Femina, v. 34, n. 2, p. 91-98, 2006.

PALUDETTI, L. R. S.; HELENO, M. G. V. The quality of life of women patients in a clinic for human reproduction. Psicólogo informação, v. 16 n. 16, p. 163-177, 2012.

PEREIRA, D. H. M. A história da reprodução humana no Brasil. Femina, v. 39, n. 2, p. 61, 2011.

RUTSTEIN, S. O.; SHAH, I. H. Infecundity, infertility, and childlessness in developing countries. DHS Comparative Reports 9. Geneva: World Health Organization, 2004.

SANTOS, I. S.; UGÁ, M. A. D.; PORTOS, M. The public-private mix in the brazilian health system: financing, delivery and utilization of health services. Ciência e Saúde Coletiva, v. 13, n. 5, p. 14311440, 2008.

SAMRSLA, M. et al. Expectativa de mulheres à espera de reprodução assistida em hospital público do DF: Estudo Bioético. Revista da Associação Médica Brasileira, v. 53, n. 1, p. 47-52, 2007.

SOUZA, E, M.; GRUNDY, E. Promoção da saúde, epidemiologia social e capital social: interrelações e perspectivas para a saúde pública. Cadernos de Saúde Pública, v. 20, n. 5, p. 1354-1360, 2004.

SOUZA, M. C. B.; VITORINO, R, J. A abordagem do casal infértil. Femina, v. 36, n. 10, p. 603609, 2008.

SHEFI, S. H. A. I; TUREK, P. A. U. L. J. Definition and current evaluation of subfertile men. International Brazilian Journal of Urology, v. 32, n. 4, p. 385-397, 2006.

SHARLIP, I. D. et al. Best practice policies for male infertility. Fertility and sterility, v. 77, n. 5, p. 873-888, 2002.

VAN, B. F.; BOS, H. M. W. The social and cultural consequences of being childless in poor-resource areas. Factore, Views e Vision is ObGyn, v. 1, n. 2, p. 106-121, 2009. 
WORLD HEALTH ORGANIZATION. Mother or nothing: the agony of infertility. News - WHO Bulletin, v. 88, n. 12, p. 877-953, 2010a.

WORLD HEALTH ORGANIZATION. Laboratory Manual for the Examination and Processing of Human Semen. 5. ed. Geneva, 2010b.

YIN, M. L. Y. Aspectos psicológicos da esterilidade feminina. 1987. p. 150. Dissertação (Mestrado em Psicologia Clínica) - Pontifícia Universidade Católica de São Paulo, São Paulo, 1987. 